\title{
Photon density of states for deformed surfaces
}

\author{
T Emig ${ }^{1}$ \\ ${ }^{1}$ Institut für Theoretische Physik, Universität zu Köln, Zülpicher Straße 77, \\ D-50937 Köln, Germany \\ E-mail: te@thp.uni-koeln.de
}

\begin{abstract}
A new approach to the Helmholtz spectrum for arbitrarily shaped boundaries and a rather general class of boundary conditions is introduced. We derive the boundary induced change of the density of states in terms of the free Green's function from which we obtain both perturbative and non-perturbative results for the Casimir interaction between deformed surfaces. As an example, we compute the lateral electrodynamic Casimir force between two corrugated surfaces over a wide parameter range. Universal behavior, fixed only by the largest wavelength component of the surface shape, is identified at large surface separations. This complements known short distance expansions which are also reproduced.

PACS numbers: 42.25.Fx, 03.70.+k, 12.20.-m, 42.50.Ct
\end{abstract}

Submitted to: J. Phys. A: Math. Gen.

\section{Introduction}

Casimir interactions can be viewed as a consequence of a change in the photon spectrum under a relative displacement of the interacting objects. For two parallel ideal metallic plates the change in the photon density of states (DOS) with the plate distance $H$ is wellknown [1. It is peaked close to the characteristic frequency $\omega \sim c / H$ which provides the dominant contribution to the interaction. However, even for simple geometries like a plate and a sphere no exact expression for the change in the DOS is available. The reason for that is the difficulty to compute the distribution of eigenfrequencies of the Helmholtz wave equation in arbitrary geometries. This is inherently linked to the problem of chaotic (quantum) billiards [2]. For Casimir interactions the natural question arises to what extent the force characterizes the shape of the interacting objects and vice versa. There is a long debate about the existence of a repulsive Casimir interaction between ideal metals which was mainly initiated by the positivity of the Casimir energy of a single infinitesimal thin sphere of ideal metal [3]. So far, no example of a repulsive force between two separated ideal metallic bodies has been found.

During the last decades a number of approximative methods have been developed to determine the Casimir interaction in more complicated geometries. These include 
the proximity and pairwise additivity approximations [4], semiclassical approaches [5], multiple scattering expansions [1] and, more recently, a simple to implement optical approach [6] which is however limited to short distances. Despite the usefulness of these approaches in certain limits, reliable results for the entire range of energies (or distances) are not known in general. Here I shall present a formula for the density of states in terms of the free space Green's function which is evaluated at the (ideal metallic) surfaces only. This formula will be used to compute the DOS for two deformed surfaces both perturbatively in the deformations and also exactly with the aid of a previously developed numerical method for periodic surfaces [7]. As an application, we study the lateral force between periodic surfaces. It is found that the surface's shape can be deduced from the force at short distances whereas at large separations universality prevails.

\section{Trace formula for the photon spectrum}

For geometries with a translational invariant direction the electromagnetic field splits into transverse electric (TE) and magnetic (TM) modes. Both modes are described by a scalar field which fulfills either Dirichlet or Neumann boundary conditions on the ideal metal surfaces. Hence, we consider in the following the scalar field Helmholtz equation

$$
\left(\nabla^{2}+k^{2}\right) \phi(\mathbf{x})=0
$$

The relevant quantity for the Casimir interaction is the change $\delta \rho(k)=\rho(k)-\rho_{\infty}(k)$ in the DOS caused by moving the surfaces from infinity to the finite distance for which one is interested in the Casimir energy. Hence $\rho_{\infty}(k)$ is the DOS for infinitely separated surfaces. The relevant finite part of the zero-point energy is then given by the sum of $k \delta \rho(k)$ over $k$. It is more convenient to work in Euclidean space by performing a Wick rotation to imaginary frequencies. With $\delta \tilde{\rho}\left(q_{0}\right)=-\delta \rho\left(i q_{0}\right)$ the Casimir energy becomes

$$
\mathcal{E}=\frac{\hbar c}{2} \int_{0}^{\infty} d q_{0} q_{0} \delta \tilde{\rho}\left(q_{0}\right) .
$$

In order to derive the DOS we start with the 4-dimensional Euclidean action

$$
S=\frac{1}{2} \int d^{4} X(\nabla \phi)^{2}
$$

Using a path-integral formulation with delta-functions enforcing the boundary conditions on the surfaces $S_{\alpha}$ [8], one obtains the two-point correlation function in the presence of boundaries [9]. With the free space Green's function $G_{0}\left(\mathbf{x}, \mathbf{x}^{\prime} ; q_{0}\right)=$ $e^{-q_{0}\left|\mathbf{x}-\mathbf{x}^{\prime}\right|} /\left(4 \pi\left|\mathbf{x}-\mathbf{x}^{\prime}\right|\right)$, the modified correlations $G\left(\mathbf{x}, \mathbf{x}^{\prime} ; q_{0}\right)=\left\langle\phi_{q_{0}}(\mathbf{x}) \phi_{-q_{0}}\left(\mathbf{x}^{\prime}\right)\right\rangle$ in the presence of boundaries is given by

$$
\begin{aligned}
G\left(\mathbf{x}, \mathbf{x}^{\prime} ; q_{0}\right) & -G_{0}\left(\mathbf{x}, \mathbf{x}^{\prime} ; q_{0}\right)=-\sum_{\alpha \beta} \int d \mathbf{u} d \mathbf{u}^{\prime} G_{0}\left(\mathbf{x}, \mathbf{s}_{\alpha}(\mathbf{u}) ; q_{0}\right) \\
& \times \mathcal{M}_{\alpha \beta}^{-1}\left(\mathbf{u}, \mathbf{u}^{\prime} ; q_{0}\right) G_{0}\left(\mathbf{x}^{\prime}, \mathbf{s}_{\beta}\left(\mathbf{u}^{\prime}\right) ; q_{0}\right)
\end{aligned}
$$


where $\mathcal{M}_{\alpha \beta}^{-1}\left(\mathbf{u}, \mathbf{u}^{\prime} ; q_{0}\right)$ is the functional inverse of $\mathcal{M}_{\alpha \beta}\left(\mathbf{u}, \mathbf{u}^{\prime} ; q_{0}\right)=G_{0}\left(\mathbf{s}_{\alpha}(\mathbf{u}), \mathbf{s}_{\beta}\left(\mathbf{u}^{\prime}\right) ; q_{0}\right)$ with the $3 \mathrm{D}$ vectors $\mathbf{s}_{\alpha}(\mathbf{u})$ denoting the positions of surface $S_{\alpha}$ as a function of the $2 \mathrm{D}$ coordinate $\mathbf{u}$. This result applies to Dirichlet boundary conditions. For the Neumann case $G_{0}$ has to be replaced by $\partial_{\mathbf{n}_{\alpha}(\mathbf{u})} G_{0}\left(\mathbf{x}, \mathbf{s}_{\alpha}(\mathbf{u}) ; q_{0}\right)$ on the right-hand side of Eq. (4) and $\mathcal{M}_{\alpha \beta}\left(\mathbf{u}, \mathbf{u}^{\prime} ; q_{0}\right)=\partial_{\mathbf{n}_{\alpha}(\mathbf{u})} \partial_{\mathbf{n}_{\beta}\left(\mathbf{u}^{\prime}\right)} G_{0}\left(\mathbf{s}_{\alpha}(\mathbf{u}), \mathbf{s}_{\beta}\left(\mathbf{u}^{\prime}\right) ; q_{0}\right)$ with $\partial_{\mathbf{n}_{\alpha}}$ the normal derivative at the surface $S_{\alpha}$. Next we make use of the property that the trace of Green's function yields the DOS. In the Euclidean formulation this relation reads

$$
\rho\left(i q_{0}\right)=\frac{2 q_{0}}{\pi} \int d^{3} \mathbf{x} G\left(\mathbf{x}, \mathbf{x} ; q_{0}\right) .
$$

Here the spatial integration extends over all inner and other regions of the surfaces. Since Eq. (41) holds in any spatial region, we obtain the change in the DOS by integrating the right-hand side of that equation with $\mathbf{x}=\mathbf{x}^{\prime}$ running over the entire 3D space. The integration is easy to perform in momentum space since $\mathbf{x}$ and $\mathbf{x}^{\prime}$ occur as arguments of the free Green's function in Eq. (4). Using for the Fourier transformed propagator $\partial / \partial q_{0}\left(q_{0}^{2}+q^{2}\right)^{-1}=-2 q_{0}\left(q_{0}^{2}+q^{2}\right)^{-2}$, one obtains after $\mathbf{x}$-integration both for $\mathcal{M}$ and $\mathcal{M}_{\infty}$ the final trace formula

$$
\delta \tilde{\rho}\left(q_{0}\right)=-\frac{1}{\pi} \frac{\partial}{\partial q_{0}} \operatorname{Tr} \ln \left(\mathcal{M} \mathcal{M}_{\infty}^{-1}\right)
$$

The trace runs over the $2 \mathrm{D}$ coordinates $\mathbf{u}$ and the surface indices $\alpha$. An advantage of this formula is its independence of surface self-energies which diverge in the limit of ideal metals but are irrelevant for the interaction. It is important to note that this formula can be applied in any spatial dimension and also to other types of boundary conditions which can be implemented via delta-functions in the path integral [10].

\section{Density of states}

In this section we will apply the trace formula of Eq. (6) to two deformed but on average parallel plates. In the first part we study (in amplitude and curvature) small deformations of general shape (corresponding to the large distance limit) by perturbation theory. Non-perturbative numerical results for the DOS of periodically corrugated surfaces with edges at closer distances are presented in the second part.

\subsection{Perturbation theory for arbitrarily deformed surfaces}

Let us consider two parallel surfaces with small deformations which are parametrized by $\mathbf{s}_{1}(\mathbf{u})=\left(\mathbf{u}, h_{1}(\mathbf{u})\right)$ and $\mathbf{s}_{2}(\mathbf{u})=\left(\mathbf{u}, H+h_{2}(\mathbf{u})\right)$ where the deformation fields $h_{\alpha}$ are small compared to the mean distance $H$. We are interested in the deformation induced modification of the flat surface DOS

$$
\delta \tilde{\rho}_{0}\left(q_{0}\right)=\frac{q_{0}}{2} \log \left(1-e^{-2 q_{0} H}\right) A
$$


where $A$ is the surface area. Expanding the kernel $\mathcal{M}=\mathcal{M}_{0}+\delta \mathcal{M}$ with $\mathcal{M}_{0}$ the kernel for flat surfaces and $\delta \mathcal{M}$ the correction due to the deformations, one finds for the change of the DOS

$$
\delta \tilde{\rho}\left(q_{0}\right)=-\frac{1}{\pi} \operatorname{Tr}\left[\log \mathcal{M}_{0}+\log \left(1+\mathcal{M}_{0}^{-1} \delta \mathcal{M}\right)\right]-" H \rightarrow \infty^{\prime \prime},
$$

where the contribution for $H \rightarrow \infty$ has to be subtracted. Expanding the logarithm to second order in the deformations $h_{\alpha}(\mathbf{u})$, the change of the DOS can be written as

$$
\begin{aligned}
\delta \tilde{\rho}\left(q_{0}\right) & =\delta \tilde{\rho}_{0}\left(q_{0}\right)-\frac{1}{4 \pi^{2}} \frac{q_{0}^{3}}{\sinh ^{2}\left(q_{0} H\right)} \int_{\mathbf{u}}\left[h_{1}^{2}(\mathbf{u})+h_{2}^{2}(\mathbf{u})\right] \\
& +\int_{\mathbf{u}, \mathbf{u}^{\prime}}\left\{\frac{1}{2} \sum_{\alpha} K_{\|}\left(q_{0} ; \mathbf{u}-\mathbf{u}^{\prime}\right)\left[h_{\alpha}(\mathbf{u})-h_{\alpha}\left(\mathbf{u}^{\prime}\right)\right]^{2}\right. \\
& \left.+\sum_{\alpha \neq \beta} K_{\times}\left(q_{0} ; \mathbf{u}-\mathbf{u}^{\prime}\right) h_{\alpha}(\mathbf{u}) h_{\beta}\left(\mathbf{u}^{\prime}\right)\right\}
\end{aligned}
$$

where $\delta \tilde{\rho}_{0}$ is given by Eq. (17). The kernels of the non-local contributions can be expressed in terms of the free Green's function. In the following we will focus due to limitations in space on Dirichlet conditions; the Neumann case has the form of Eq. (9) with however different kernels. Defining the series

$$
\begin{aligned}
& R_{\text {even }}\left(q_{0} ; u\right)=\sum_{n=1}^{\infty} \frac{G_{0}\left(\sqrt{u^{2}+(2 n H)^{2}} ; q_{0}\right)}{(2 n)^{2}}, \\
& R_{\text {odd }}\left(q_{0} ; u\right)=\sum_{n=1}^{\infty} \frac{G_{0}\left(\sqrt{u^{2}+((2 n-1) H)^{2}} ; q_{0}\right)}{(2 n-1)^{2}}
\end{aligned}
$$

the kernels can be written as

$$
\begin{aligned}
& K_{\|}\left(q_{0} ; \mathbf{u}\right)=-\frac{8}{\pi} \partial_{q_{0}}\left[\left(\frac{1}{u} \partial_{u} G_{0}\left(u ; q_{0}\right)+\partial_{H}^{2} R_{\text {even }}\left(q_{0} ; u\right)\right) \partial_{H}^{2} R_{\text {even }}\left(q_{0} ; u\right)\right] \\
& K_{\times}\left(q_{0} ; \mathbf{u}\right)=-\frac{8}{\pi} \partial_{q_{0}}\left[\partial_{H}^{2} R_{\text {odd }}\left(q_{0} ; u\right)\right]^{2} .
\end{aligned}
$$

The contributions to the kernels can be viewed as multiple scattering paths between flat surface positions. The kernel $K_{\|}$has only contributions from an even number of paths crossing the gap, connecting two positions on the same surface, see Fig. 1(a). The first factor $\sim \partial_{u} G_{0}$ in $K_{\|}$is represented as a non-crossing paths. An odd number of crossing paths yields the kernel $K_{\times}$, see Fig. 1(b). Each path is associated with the free Green's function which is reduced by a factor which is the inverse squared number of gap crossings, cf. Eq. (10).

\subsection{Non-perturbative treatment of periodic surfaces}

The above perturbative treatment is limited to small and smooth deformations. However, the trace formula turns out to be useful also in the case of surfaces with edges, and at large deformation amplitudes. Recently, a numerical tool has been 


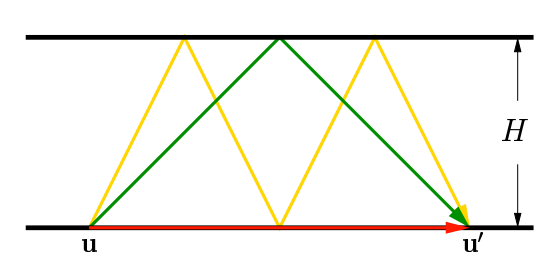

(a)

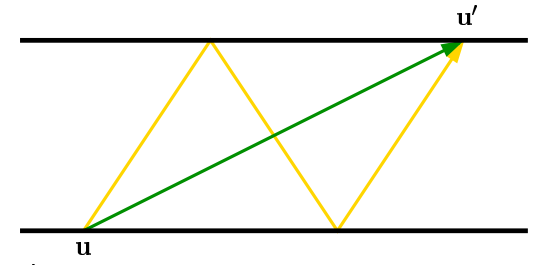

(b)

Figure 1. Graphical representation of the contributions to the kernels $K_{\|}$(a) and $K_{\times}$ (b), cf. Eq. (111).

developed to evaluate the Casimir force for periodic surface deformations [7]. Here we shall demonstrate that same approach can be also applied to the DOS. Specifically, we consider uni-axial rectangular surface profiles shown in Fig. 3(a). In this section we restrict the analysis to no lateral shift, $b=0$. By Fourier transforming $\mathcal{M}_{\alpha \beta}\left(\mathbf{u}, \mathbf{u}^{\prime} ; q_{0}\right)$ with respect to $\mathbf{u}$ and $\mathbf{u}^{\prime}$ one can employ the periodicity of the surface profiles (along the $x_{1}$-direction) which leads to the representation

$$
\begin{aligned}
& \tilde{\mathcal{M}}_{\alpha \beta}\left(\mathbf{p}, \mathbf{q} ; q_{0}\right)=(2 \pi)^{2} \delta\left(p_{2}+q_{2}\right) \\
& \times \sum_{m=-\infty}^{\infty} \delta\left(p_{1}+q_{1}+2 \pi m / \lambda\right) N_{m}^{\alpha \beta}\left(q_{1}, q_{2} ; q_{0}\right),
\end{aligned}
$$

which defines the $2 \times 2$ matrices $N_{m}$ that can be computed analytically and are given in [7] for the present geometry. $\tilde{\mathcal{M}}$ can be transformed to block-diagonal form so that each block couples only waves whose momenta differ from a given Bloch momentum by integer multiples of $2 \pi / \lambda$. This decomposition can be used to define the function

$$
g\left(q_{1}, q_{2} ; q_{0}\right)=\operatorname{tr}\left(B^{-1} \partial_{q_{0}} B-B_{\infty}^{-1} \partial_{q_{0}} B_{\infty}\right)
$$

where the trace runs over all discrete indices of the matrix $B_{k l}^{\alpha \beta}\left(q_{1}, q_{2} ; q_{0}\right)=N_{k-l}^{\alpha \beta}\left(q_{1}+\right.$ $\left.2 \pi l / \lambda, q_{2} ; q_{0}\right)$, and $B_{\infty}$ is the analog of $\mathcal{M}_{\infty}$, i.e., $B$ for $H \rightarrow \infty$. For practical computations we take $k, l=-M, \ldots, M$ with an integer cutoff $M$ which should be taken to infinity. The change of the DOS can then be expressed as

$$
\delta \tilde{\rho}\left(q_{0}\right)=-\frac{A}{4 \pi^{3}} \int_{-\infty}^{\infty} d q_{2} \int_{0}^{2 \pi / \lambda} d q_{1} g\left(q_{1}, q_{2} ; q_{0}\right) .
$$

The following results for electromagnetic fluctuations are based on the application of this formula to Dirichlet (TM modes) and Neumann (TE modes) boundary conditions where the inversion of $B$, the trace in Eq. (13) and the integration are performed numerically.

Fig. 2 displays the change of the DOS separately for TM and TE modes at the fixed distance $H=10 a$ and $\lambda$ ranging from $a$ to $100 a$. Plotted is also the analytic expression for flat plates at the same mean distance and at the reduced distance $H-2 a$. An expected feature of these results is that the dominant contribution to the change of the DOS comes from frequencies $q_{0} \sim H^{-1}$. For small modulation lengths $\lambda \ll H$ 

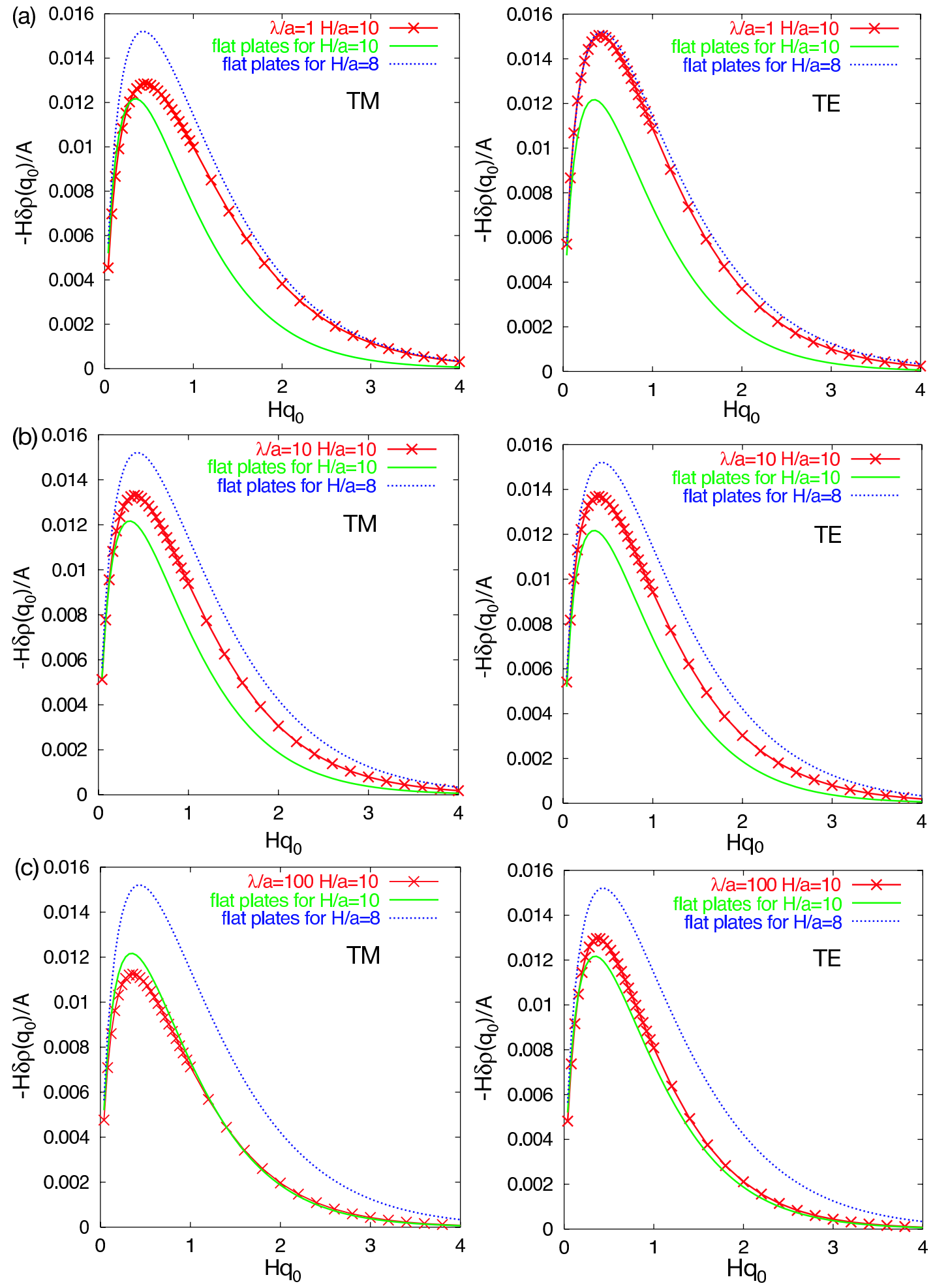

Figure 2. Change of the density of states $\delta \rho\left(q_{0}\right)$ per unit area for TM and TE modes with parameters $H=10 a$ and $\lambda / a=1$ (a), 10 (b), 100 (c). The results were obtained with a cutoff of $M=13$. (For scaling of the $\left(H q_{0}\right)$-axis, $H=10 a$.) 
one can expect that for $q_{0} \lesssim \lambda^{-1}$ the modes cannot probe the narrow valleys of the surface, and the DOS should be well described by the result for flat plates at a reduced distance $H-2 a$. While this is fully confirmed for TE modes, the magnetic modes show an unexplained deviation at sufficiently small $q_{0}$, see Fig. 3(a). For $\lambda \sim H$, Fig. 3(b), no significant differences between the two types of modes are observed, and the change of the DOS is in between the result for flat surfaces at distances $H$ and $H-2 a$, respectively. For large $\lambda \gg H$, Fig. 3(c), there is a low density of edges, and one can expect the DOS for flat plates at distance $H$ to be a good approximation. Such behavior is indeed observed with a small decrease for TM and a small increase for TE modes close to the peak.

\section{Universality of the lateral Casimir force}

Finally, we use the previous results to study the Casimir force for the geometry in Fig. 31(a) which emerges from the variation of the zero-point energy with the shift $b$. The energy can be obtained from the DOS via Eq. (2). Without giving further details we note that the lateral force $F_{l}=-\partial \mathcal{E} / \partial b$ can be obtained by computing directly the derivative of the matrices $N_{m}^{\alpha \beta}$ in Eq. (12) with respect to $b$. The results are shown as a function of the surface distance for a fixed $b=\lambda / 4$ in Fig. 3(c) and at a fixed $H=10 a$ as a function of $b$ in Fig. 3(d). Let us compare to approximative methods for small $H$. The proximity force approximation [4] yields the lateral force $F_{\mathrm{PFA}}=$ $A\left[2 \mathcal{E}_{0}(H)-\mathcal{E}_{0}(H-2 a)-\mathcal{E}_{0}(H+2 a)\right] / \lambda$ for $0<b<\lambda / 2$ with $\mathcal{E}_{0}(H)=-\left(\pi^{2} / 720\right) \hbar c / H^{3}$, which changes sign at $b=\lambda / 2$ discontinuously. The pairwise summation (PWS) of Casimir-Polder potentials $U(r)=-(\pi / 24) \hbar c / r^{7}$ is often naively applied to metals, using an "corrected" amplitude as to reproduce the correct result for flat ideal metal plates [4]. For the present geometry $F_{\text {PWs }}$ can be computed by a simple numerical integration. The two approximations are also shown in Fig. [3(c),(d). For small $\delta$, both approximations agree and match our results. Beyond $\delta \gtrsim \lambda / 20$ the PFA starts to fail since it does not capture the exponential decay of $F_{\text {lat }}$ for increasing $\delta$. The PWS approach has a slightly larger validity range [cf. Fig $[3(b)]$ and reproduces the exponential decay. However it deviates by at least one order of magnitude from $F_{\text {lat }}$ for $\delta \gtrsim 2.5 \lambda$.

At large $\delta \gtrsim \lambda$ the lateral force shows universal behavior in the form of approaching a limiting form which is independent of the detailed shape of the surface corrugation. The universal limit is given by the lateral force for a sinusoidally shaped surface (with amplitude $a_{0}$ and wavelength $\lambda$ ) [1],

$$
F_{\mathrm{pt}}=\frac{8 \pi^{3} \hbar c}{15} \frac{a_{0}^{2} A}{\lambda^{5} H} \sin \left(\frac{2 \pi}{\lambda} b\right) e^{-2 \pi H / \lambda},
$$

which is expected to hold for $a_{0} \ll \lambda$. Using the lowest harmonic of the present rectangular geometry, corresponding to $a_{0}=4 a / \pi$, we find indeed excellent agreement between $F_{\mathrm{pt}}$ and our results for the geometry of Fig 3(a) for large $\delta \gtrsim \lambda$, see Fig (3) (c).

For the dependence on $b$, see Fig. 3(d), three regimes can be identified. For $\lambda \gg H$,

the force profile resembles almost the rectangular shape of the surfaces, and the PWS 

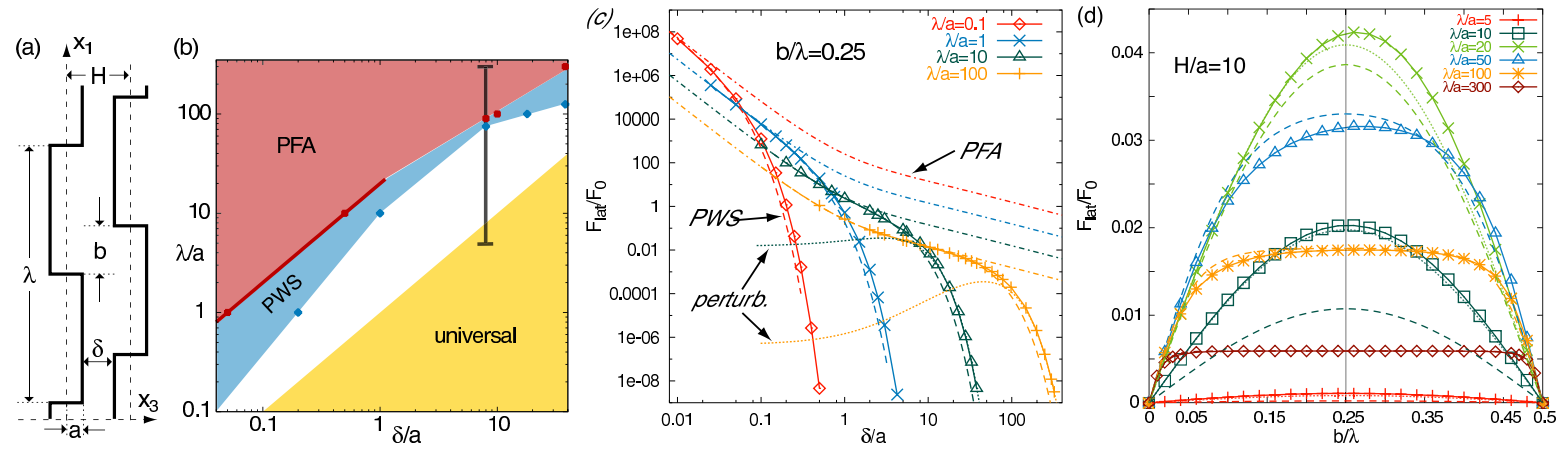

Figure 3. (a) Two plate geometry. (b) Approximate validity ranges of PFA and PWS, and the universal parameter range. The vertical bar marks the parameter range for $\lambda / a$ in (d). (c) Lateral force (in units of the normal force $F_{0}(d)$ between flat surfaces). Shown are also the PFA (dash-dotted), PWS (dashed) and the universal limit $F_{\mathrm{pt}}$ (dotted). (d) Shape dependence of the lateral force. The dashed and the dotted curves are the PWS and the full perturbative result of [1], respectively.

approach yields consistent results. For decreasing $\lambda$, yet larger than $H$, the force profile becomes asymmetric with respect to $b=\lambda / 4$ and more peaked, signaling the crossover to the universal regime for $\lambda \lesssim H$ where the force profile becomes sinusoidal. We note that the PWS approach fails to predict the asymmetry of the force profile, and the PFA even predicts no variation for $0<b<\lambda / 2$.

\section{Acknowledgments}

Support through the Emmy-Noether Program of the DFG under grant No. EM70/2 is acknowledged.

\section{References}

[1] R Balian and B Duplantier, 1978 Ann. Phys. (N.Y.) 112165

[2] P Cvitanović et al., Chaos: Classical and Quantum (Niels Bohr Institute, Copenhagen, 2003), chaosBook.org

[3] T Boyer, 1974 Phys. Rev. A 92078

[4] M Bordag, U Mohideen, and V M Mostepanenko, 2001 Phys. Rep. 3531

[5] M Schaden and L Spruch, 1998 Phys. Rev. A 58935

[6] R L Jaffe and A Scardicchio, 2004 Phys. Rev. Lett. 92070402

[7] R Büscher and T Emig, 2004 Phys. Rev. A 69062101

[8] H Li and M Kardar, 1992 Phys. Rev. A 466490

[9] R Büscher and T Emig, 2005 Phys. Rev. Lett. 94133901

[10] R Büscher and T Emig, 2004 Nucl. Phys. B696 468

[11] T Emig, A Hanke, R Golestanian, and M Kardar 2003 Phys. Rev. A 67022114 